\title{
O Impacto das Transferências Constitucionais sobre os Gastos dos Municípios Brasileiros
}

\section{The Impact of Constitutional Transfers on Expenses of Brazilian Municipalities}

\author{
Rodolfo Ferreira Ribeiro da Costa* \\ Luiz Ivan de Melo Castelar**
}

\begin{abstract}
Resumo: Em países onde o sistema de governo possui um caráter descentralizado, observa-se uma forte dependência de recursos provenientes das entidades superiores da federação por parte das esferas inferiores da administração pública. No Brasil, tal fato é confirmado pelo fato das transferências intergovernamentais representarem, em média, um volume superior a $88 \%$ das receitas totais. Apesar da sua importância na composição das receitas das gestões municipais, o financiamento da atividade pública via sistema de repasses intergovernamentais pode levar a prejuízos na gestão municipal. Dentre os principais transtornos que um regime federativo pode sofrer mediante o mecanismo de financiamento das esferas inferiores do governo via transferências constitucionais, tem-se a realização de despesas sem compromisso com uma gestão de recursos equilibrada. Diante desse cenário, o objetivo deste trabalho é verificar a ocorrência de práticas condizentes com definição do efeito flypaper. A descrição dessa relação é feita por meio da técnica de vetores autorregressivos e do modelo de regressões quantílicas, ambos em suas versões para dados em painel. A amostra utilizada é composta por informações sobre a arrecadação, PIB, população, transferências correntes e gastos para 5.293 municípios brasileiros entre 1999 e 2009, extraídos da Secretaria do Tesouro Nacional e do Instituto Brasileiro de Geografia e Estatística. Os resultados destacam que não foram observadas condições que confirme a prática do efeito flypaper pela administração pública municipal no Brasil. Por fim, verifica-se que os efeitos das transferências sobre as despesas dos municípios brasileiros não são expressos somente para uma análise geral, mas sim, para diferentes quantis da distribuição.
\end{abstract}

Palavras-chave: Transferências. Flypaper. PVAR. Quantis.

\begin{abstract}
In countries where the government system has a decentralized character observes a strong dependence on resources from the higher authorities of the federation, by the lower spheres of government. In Brazil, this fact is confirmed by the fact that intergovernmental transfers represent, on average, a volume higher than $88 \%$ of total revenues. Despite its importance in the composition of revenues of municipal administrations, funding of public system via intergovernmental transfers

\footnotetext{
* Doutor em Economia pela Universidade Federal do Ceará (UFC). Professor adjunto do Departamento de Economia (DEC) e do Programa de Pós-Graduação em Economia (PPECO) da Universidade do Estado do Rio Grande do Norte (UERN). E-mail: rodolfofrc@yahoo.com.br

** Pós-doutor pela University of Aberdeen. Doutor em Econometria pela University of Florida. Professor titular do Departamento de Teoria Econômica (DTE) e do Curso de Pós-Graduação em Economia (Caen) da Universidade Federal do Ceará (UFC). E-mail: lume1250@yahoo.com.br
} 
can lead to losses in municipal management. The main inconvenience than a federal system may suffer through the funding mechanism of the lower spheres of government through constitutional transfers has to incur expenses without commitment to a balanced resource management. In this scenario, the objective of this work is to verify the occurrence of practices consistent with the definition of the flypaper effect. The description of this relationship will give through the technique of Vector Autoregressive model and quantile regressions, in both its versions for panel data. The sample consists of information on the collection, GDP, population, expenditure and current transfers to 5,293 Brazilian municipalities between 1999 and 2009, extracted from the National Treasury and the Brazilian Institute of Geography and Statistics. The results highlight that there were no conditions to confirm the practice of flypaper effect by municipal government in Brazil. Finally, it was found that the effects of transfers on the expenditure of municipalities are not expressed only a general analysis, but for different quantiles of the distribution.

Keywords: Transfers. Flypaper. PVAR. Quantile.

JEL Classification: H70; H77; C33.

\section{1 lntrodução}

Um sistema de governo descentralizado é caracterizado por uma forte dependência dos entes inferiores em relação aos níveis mais elevados. Seja com respeito à formação de receitas a partir de sua própria base tributária, a qual se apresenta de forma bastante limitada, ou pelo conjunto de necessidades locais, esse tipo de estrutura tende a apresentar desequilíbrios fiscais e regionais.

Para que as esferas inferiores do governo consigam praticar um orçamento de forma equilibrada e condizente com a estrutura de custos existente, faz-se necessário a realização de transferências intergovernamentais. No Brasil, o sistema de repasse que visa compatibilizar as diferenças regionais na provisão de bens públicos locais e a ocorrência de desequilíbrios fiscais dos governos municipais têm como principais componentes o Fundo de Participação dos Municípios (FPM), por parte do governo federal, e as cotas de ICMS e IPVA, alocadas pelo governo estadual. Entre 1999 e 2009, essas fontes correspondem, em média, a 88,79\% das receitas correntes dos municípios brasileiros.

Apesar de apresentar-se de forma imprescindível para as ações de um regime federativo, as transferências constitucionais podem incentivar um comportamento imprudente por parte dos gestores municipais. Em federações onde o principal componente das receitas correntes são os repasses intergovernamentais, pode-se observar uma realização de despesas sem compromisso com uma gestão de recursos equilibrada.

A literatura tem destacado o caráter prejudicial das transferências sobre o comportamento dos gastos municipais. A postura de elevação das despesas a níveis superiores ao apresentado pelo volume de recursos captados junto às esferas 
superiores do governo, ação essa designada como efeito flypaper, pode ser vista em Gramlich e Galper (1973), Gramlich (1977), Courant, Gramlich e Rubinfield (1979), Oates (1979), Fisher (1982), Filimon et al. (1982), Strumpf (1998) e Cossío e Carvalho (2001).

A irresponsabilidade fiscal das administrações subnacionais, expressa na expansão das despesas públicas que podem causar desequilíbrios orçamentários, é uma questão que necessita ser diagnosticada e fiscalizada, de forma que a gestão dos recursos públicos promova a eficiência, equidade, crescimento e bem-estar para a população.

Dessa forma, o objetivo deste trabalho é verificar a ocorrência do efeito flypaper. Para a realização deste trabalho foram coletados dados de 5.293 municípios, referentes à arrecadação própria, despesas totais, transferências correntes, população e PIB, com periodicidade anual e correspondentes ao intervalo de tempo entre 1999 e 2009. A metodologia utilizada fundamenta-se no modelo do eleitor mediano num ambiente de incerteza e a estratégia empírica adotada baseia-se na técnica de vetores autorregressivos e no modelo de regressões quantílicas, ambos em suas versões para dados em painel, propostas por Holtz (1988) e Koenker (2004), respectivamente. A primeira dessas técnicas propiciará o acompanhamento dinâmico e geral para as referidas relações, enquanto que a última evidenciará uma análise estática e mais detalhada da distribuição. A junção dessas técnicas ainda permitirá verificar a possibilidade de diferenças entre o efeito geral e aqueles observados em distintos pontos da distribuição.

Além desta seção introdutória, o trabalho apresenta mais quatro seções. A segunda discute o modelo teórico do eleitor mediano com considerações de incerteza com foco na caracterização do efeito flypaper e apresenta uma breve revisão empírica sobre tal fenômeno. A terceira destaca a ferramenta utilizada para verificação da prática do efeito flypaper, bem como a descrição do modelo empírico e dos dados utilizados. A quarta relata os resultados que possibilitarão identificar a ocorrência, ou não, do efeito flypaper. Por fim, a quinta expõe as considerações finais.

\section{Relação entre as Transferências Governamentais e os Gastos Públicos}

Um dos principais temas discutidos em finanças públicas diz respeito ao efeito flypaper, fenômeno observado quando os repasses intergovernamentais apresentam-se de forma mais estimulante para despesas locais do que as variações na renda. A maioria das tentativas de explicar o efeito flypaper é trabalhada a partir do modelo do eleitor mediano associado à hipótese de que os eleitores tomam suas decisões sem informação perfeita sobre como o setor público traduz seus desejos em política. 
A premissa dessa abordagem é que os modelos de escolha racional do consumidor sob certeza não podem lidar adequadamente com o conceito de ilusão fiscal, já que o eleitor representativo não possui todas as informações sobre as ações fiscais do Estado. Assim, tal corrente utiliza-se dos fundamentos da teoria de escolha do consumidor sob incerteza, introduzindo a ideia de que os eleitores tomam suas decisões com informação imperfeita, dado o comportamento estocástico apresentado pelas trasnferências constitucionais e pela composição da base de arrecadação.

\subsection{Modelo do Eleitor Mediano com Informação Imperfeita}

A análise do comportamento das despesas governamentais será realizada a partir do modelo do eleitor mediano com informação imperfeita descrito por Turnbull (1992), em que o consumidor representativo decide sobre as quantidades de bens públicos e privados que maximizam sua utilidade esperada.

O governo, através de suas receitas próprias e do recebimento de repasses intergovernamentais, é responsável por prover um conjunto de bens e serviços à população. Por simplicidade, admite-se que a produção é formada por um único bem que contempla as características idealizadas nas necessidades locais e que pode ser expressa a partir da seguinte equação:

$$
e=t(B+\theta)+A+\varepsilon
$$

em que $e$ é a oferta de bens públicos; t é a alíquota do imposto; $B$ é a capacidade tributária da jurisdição formada por $n b$, sendo $n$ o tamanho da população e $b$ a base tributária individual; $\theta$ é um componente estocástico da arrecadação, que possui média zero e variância finita; $A$ representa as transferências governamentais; e $\varepsilon$ é o termo aleatório das transferências com média zero e variância finita.

Como a cesta de consumo do o eleitor representativo é formada por bens privados e públicos, sendo a produção deste fomentada pelo Estado, admite-se que o mesmo divide seus rendimentos entre o pagamento de taxas e o consumo de bens privados. A equação de rendimentos é dada como:

$$
l=t b+x
$$

em que $l$ é a renda do eleitor; $b$ é a capacidade tributária individual; e $x$ é o consumo de bens privados.

Seja $E U(x, e)$ a utilidade esperada, então o problema do eleitor, a partir das equações 1 e 2, é definido pela escolha da alíquota ótima que maximiza sua utilidade esperada: 


$$
\max _{t} E U(l-t b, t(B+\theta)+A+\varepsilon)
$$

O problema requer o seguinte conjunto de condições, necessárias e suficientes, respectivamente, de modo que se possa garantir que a escolha da alíquota $t$ pelo consumidor seja aquela que proporcione o maior nível de utilidade possivel:

$$
\begin{gathered}
-E U_{x} b+E U_{e}(B+\theta)=0 \\
J=E U_{x x} b^{2}+E U_{e e}(B+\theta)^{2}-2 E U_{x e} b(B+\theta)<0
\end{gathered}
$$

A forma quadrática e negativa de $J$ deve-se ao formato côncavo assumido para função de utilidade esperada.

De acordo com as condições de primeira ordem do problema e assumindo que o agente representativo tenha aversão ao risco, ${ }^{1}$ verifica-se que, num ambiente com informação imperfeita, caracterizado por incerteza, a taxa marginal de substituição entre bens públicos e privados é dada pela proporção da base individual em relação à base total e um prêmio de risco pelo consumo de bens privados, como detalhado na equação 5:

$$
\frac{E U_{e}}{E U_{x}}=\frac{b}{B}-\frac{E U_{e} \theta}{E U_{x} B}
$$

Considerando a base como fixa, $\bar{B}$, e as funções de oferta de serviços públicos e de rendimentos do eleitor, expressas nas equações 1 e 2 , respectivamente, podese verificar o efeito dos repasses das esferas superiores e da renda do agente representativo sobre os gastos municipais a partir das seguintes expressões:

$$
\begin{aligned}
& \frac{d E[e]}{d l}=-B\left[-E U_{x x} b+E U_{e x}(B+\theta)\right] / J \\
& \frac{d E[e]}{d A}=1-B\left[-E U_{x e} b+E U_{e e}(B+\theta)\right] / J
\end{aligned}
$$

As equações 6 e 7 provêm da diferenciação implícita da condição de primeira ordem do problema do eleitor.

A comparação dos impactos relativos de mudanças na renda do eleitor e no montante de repasses intergovernamentais sobre as despesas municipais, que produzirá os argumentos necessários para verificação do efeito flypaper, pode ser realizada pela diferenças entre as equações 6 e 7 . Tomando $s=b / B$ e substituindo o resultado na equação 7 , temos a relação que descreverá a magnitude da diferença expressa pelo efeito renda em relaçao ao efeito transferência sobre os gastos:

1 A condição de aversão ao risco é estabelecida quando $-E U_{e} \theta=-\operatorname{cov}\left(U_{e}, \theta\right)>0$. 


$$
\begin{gathered}
\frac{d E[e]}{d l}-\frac{d E[e]}{d s A} \underset{\gtrless}{\gtrless} \\
-B\left[-E U_{x x} b+E U_{e x}(B+\theta)\right] / J \gtreqless\left(\frac{B}{b}\right)-\left(\frac{B^{2}}{b}\right)\left[-E U_{x e} b+E U_{e e}(B+\theta)\right] / J
\end{gathered}
$$

Multiplicando a expressão por $\frac{-J b}{B}$, tem-se:

$$
-\left[E U_{x x} b^{2}+E U_{e e} B^{2}-2 E U_{e x} b(B+\theta)\right] \gtreqless-J
$$

Da condição de segunda ordem do problema do eleitor mediano, expressa na equação 4, tem-se que $J=E U_{x x} b^{2}+E U_{e e}(B+\theta)^{2}-2 E U_{x e} b(B+\theta)$, logo a relação estabelecida na equação 8 somente pode ser mantida com a igualdade. Ou seja, num ambiente com incerteza não se observam impactos de forma assimétrica da renda e das transferências sobre os gastos públicos, assim não há nenhuma possibilidade do estabelecimento da prática definida pela literatura como efeito flypaper.

\subsection{Evidências sobre o Efeito Flypaper}

A existencia do efeito flypaper é bastante discutida na literatura de federalismo fiscal, principalmente através de análise empíricas das mais diversas estruturas de governo descetralizadas. Contudo, os resultados apresentados diferenciam-se de acordo com as condições impostas pela abordagem teórica.

A verificação de que as transferências intergovernamentais afetam de maneira mais significativa as decisões dos gastos públicos do que o observado para a renda média da população são apresentadas, principalmente, naqueles estudos baseados em versões do modelo do eleitor mediano em ambientes de certeza. Exemplos desse tipo de resultado podem ser vistos em Gramlich e Galper (1973), Gramlich (1977), Courant, Gramlich e Rubinfield (1979), Oates (1979), Fisher (1982), Filimon et al. (1982) e Strumpf (1998). Nesse mesmo sentido, mas com considerações em ambientes de incerteza, Cossío e Carvalho (2001) verificam que o efeito transferência é oito vezes maior do que o efeito renda, caracterizando a gestão municipal brasileira como realizadora da referida prática.

Por outro lado, estudos realizados a partir da estrutura dos modelos de ilusão fiscal verificam que a equivalência do efeito transferência-renda sobre os gastos municipais se faz presente em condições de incerteza, como destacado por Turnbull (1992). No que se refere à realidade brasileira, a não observação de 
gestões imprudentes condizente com a definição do efeito flypaper são relatadas em Cossío (1998), Nascimento (2010) e Mattos et al. (2011).

\section{Verificando os Efeitos das Transferências sobre os Gastos Municipais}

Com o intuito de analisar o impacto das transferências constitucionais sobre o comportamento das despesas dos municípios brasileiros e, assim, identificar a possibilidade de ocorrência do efeito flypaper pelas prefeituras, utilizar-se-á uma estrutura para captar o efeito geral dessa relação e outra para identificar se de fato o resultado geral é válido para alguns conjuntos de jurisdições. A realização de tal tarefa passará pelo uso da técnica de vetores autorregressivos e pelo modelo de regressões quantílicas, ambos nas suas respectivas versões para dados em painel, propostas por Holtz (1988) e Koenker (2004), respectivamente.

Dado que o modelo de vetores autorregressivos para dados em painel (PVAR) caracteriza-se como um modelo dinâmico, além de considerar todas as variáveis do sistema como endógenas, e o modelo de regressão quantílica basearse num análise estática e com variáveis exógenas, as respostas proporcionadas em cada estrutura possibilitará uma análise comparativa entre o efeito médio e aqueles observados ao longo de toda a distribuição, ou seja, a utilização das referidas técnicas indicará se o efeito flypaper ocorre ou não, em média, ou, ainda, se esse tipo de resultado só pode ser verificado em algumas classes de municípios.

\subsection{Análise Empírica e Definição dos Dados}

Objetivando verificar a ocorrência de práticas imprudentes pelos gestores dos municípios brasileiros no que tange à realização de gastos numa proporção superior ao volume de repasses que formam, em média, mais de $90 \%$ de suas receitas, utilizar-se-á uma função dos gastos do governo de acordo com o modelo do eleitor mediado apresentado por Turnbull (1992).

A função baseada na técnica de regressões quantílicas para dados em painel que destacará se o comportamento dos gastos realizados pelas prefeituras brasileiras é condizente com a definição do efeito flypaper, sendo apresentada assim:

$$
Y_{\mathrm{it}}=\alpha_{\mathrm{i}}+\beta_{1} \text { população }_{\mathrm{it}}+\beta_{2} \text { Transferências }_{\mathrm{it}}+\beta_{3} \text { PIB }_{\mathrm{it}}+\beta_{4} \text { arrecadação }_{\mathrm{it}}+\varepsilon_{\mathrm{it}}
$$

em que $Y_{\mathrm{it}}$ é gasto total do i-ésimo município no período $t$; populaçãoit é a população do i-ésimo município no período $t$; Transferências ${ }_{i t}$ são as transferências realizadas pela União e estados ao i-ésimo município no período $t ;$ PIB $_{i t}$ é o produto

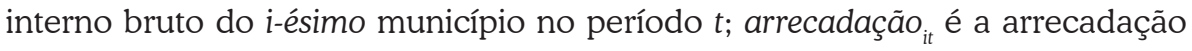


própria do i-ésimo município no período $t$; e $\varepsilon_{i t}$ é o termo de erro do i-ésimo município no período $t$.

O modelo de vetores autorregressivos para dados em painel que será utilizado é formado de acordo com Binder, Hsiao e Pesaram (2004):

$$
w_{i t}=\left(I_{m}-\Phi\right) \mu_{i}+\Phi w_{i, t-1}+\varepsilon_{i t}
$$

para $i=1,2, \ldots, n$ e $t=1,2, \ldots, T$, em que: $w_{i t}$ é um vetor $n t \times 1$ de variáveis, formado pelo gasto municipal, tamanho da população, PIB, montante de transferências repassadas pela União e estados aos municípios e pela arrecadação própria; $\Phi$ é uma matriz $m \times m$ de parâmetros; $\mu_{i}$ é um vetor $m \times 1$ de efeitos individuais; $\varepsilon_{i t}$ é o termo de erro; e $I_{m}$ é uma matriz identidade de dimensão $m \times m$.

Foram coletados dados sobre o gasto público, população, arrecadação, PIB e transferências constitucionais para 5.293 municípios brasileiros anualmente, entre 1999 e 2009. Tais informações foram extraídas do Finbra, disponibilizada pela Secretaria do Tesouro Nacional, e do IBGE.

A Tabela 1 apresenta as variáveis utilizadas, bem como sua fonte de origem. Tabela 1 - Relação, descrição e fonte das variáveis utilizadas

\begin{tabular}{l|l|c}
\hline \multicolumn{1}{c|}{ Variável } & \multicolumn{1}{c|}{ Descrição } & Fonte \\
\hline Gasto & Despesas totais & Finbra \\
Arrecadação & Arrecadação municipal & Finbra \\
População & Número de residentes & IBGE \\
PIB & Produto interno bruto & IBGE \\
Transferências & Transferências correntes & Finbra \\
\hline
\end{tabular}

Fonte: Elaboração própria.

Realizada a apresentação das funções que destacaram o comportamento dos gastos municipais de acordo com o modelo do eleitor mediano e as especificações estabelecidas pelas técnicas de vetores autorregressivos para dados em painel e regressões quantílicas para dados longitudinais, como abordado por Binder, Hsiao e Pesaram (2004) e Koenker (2004), além da descrição e apresentação das fontes primária dos dados, passa-se ao detalhamento das condições que marcam a relação entre tais variáveis. A Tabela 2 apresenta o comportamento esperado para os efeitos da população, PIB, transferências e arrecadação sobre as despesas públicas das esferas inferiores da república brasileira. 
Tabela 2 - Efeitos esperados das variáveis explicativas sobre o PIB per capita

\begin{tabular}{|c|c|}
\hline \multicolumn{2}{|c|}{ Variável dependente: gastos } \\
\hline Variável & Efeito sobre gastos \\
\hline População & + \\
\hline PIB & + \\
\hline Transferências & + \\
\hline Arrecadação & + \\
\hline
\end{tabular}

Fonte: Elaboração própria.

O sinal positivo para todos os parâmetros a serem estimados retrata bem o efeito que as variáveis que formam a demanda por serviços públicos e aquelas responsáveis pela geração de receitas, sendo a população e o PIB variáveis que representam a procura por bens e serviços públicos, enquanto que as transferências constitucionais e a arrecadação própria são as fontes do financiamento municipal.

Variações no contingente populacional e/ou na atividade econômica provocam flutuações na demanda por serviços. Por um lado, um aumento do número de residentes em um dado município requer uma variação na oferta de serviços de saúde, educação, saneamento, urbanismo, etc., tal que se faz necessária uma elevação dos gastos praticados pelas prefeituras para atender às necessidades locais. Por outro lado, o desenvolvimento da produção, seja via serviços, agropecuária ou indústria, requerem um conjunto de investimentos capazes de garantir alguns dos fatores essenciais à instalação e manutenção da atividade produtiva, quais sejam infraestrutura, qualificação da mão de obra, centros de coleta de resíduos industriais, serviços ambulatoriais, etc. Portanto, espera-se que o crescimento do PIB provoque uma elevação nos gastos dos municípios.

Como as receitas municipais são formadas por repasses do Estado e da União, bem como do seu esforço sobre a sua própria base tributária, é plausível supor que, para um maior volume de recursos, os governos locais sejam capazes de providenciar uma maior oferta de serviços. Portanto, acredita-se que uma variação nas receitas promova uma variação nos gastos na mesma direção.

\section{Resultados}

Com o objetivo de identificar o efeito flypaper nas prefeituras brasileiras, foram utilizadas as técnicas de vetores autorregressivos para dados em painel, o que proporciona a observação do comportamento geral dos gastos das esferas municipais a partir de um choque nas transferências, no PIB, na população e na arrecadação, e de regressões quantílicas para dados em painel, que identifica a 
movimento de tal relação de forma mais definida ao longo da distribuição. Tais estruturas proporcionam o acompanhamento dos efeitos do tamanho da população e da arrecadação própria sobre as despesas municipais.

Através dessas metodologias é possível verificar a existência do efeito flypaper para o conjunto de 5.293 municípios brasileiros que se fazem presente na amostra utilizada. Além disso, relatar-se-á se tal fenômeno está presente em estratos formados a partir do volume de gastos realizados pelas prefeituras.

Assim, os resultados que seguem evidenciam se os repasses constitucionais provocam um efeito expansivo nos gastos das esferas inferiores de governo numa proporção superior à observada pelos mesmos de uma forma geral, ou se esse fato somente pode ser observado para uma ou mais classes de jurisdições.

A Tabela 3 apresenta as funções impulso-resposta geradas a partir de um PVAR(1), com as séries sendo caracterizadas como um processo I(1). A utilização do modelo autorregressivo com uma única defasagem deve-se aos critérios utilizados por Love e Zecchino (2006). Ainda, a justificativa para o uso das variáveis em primeira diferença deve-se aos resultados dos testes de Harris (1999), Hadri (2000), Breitung (2001), Choin (2005), Levin et al. (2002) e Im et al. (2003), que caracterizaram as séries como integradas de ordem 1 .

Tabela 3 - Função impulso: resposta para o PVAR(1)
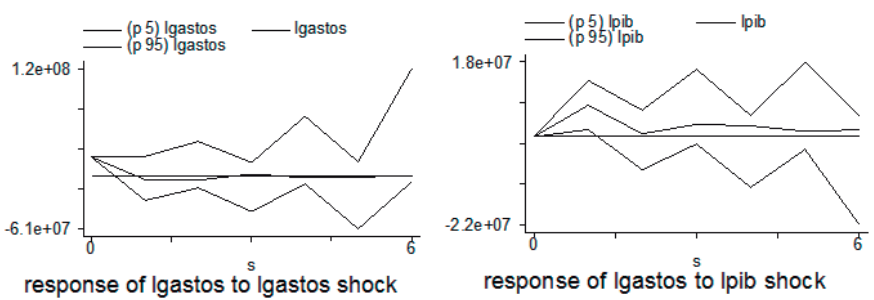

response of Igastos to Ipib shock

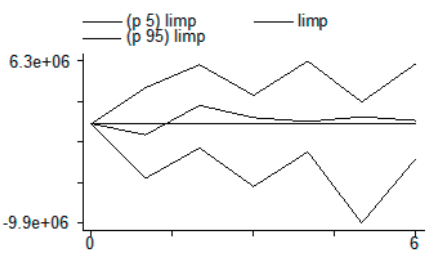

response of Igastos to limp shock

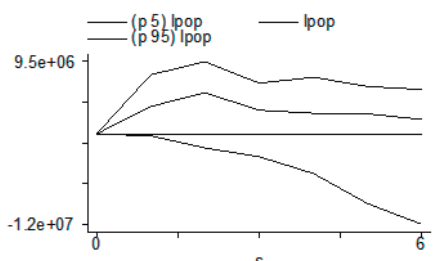

response of Igastos to Ipop shock

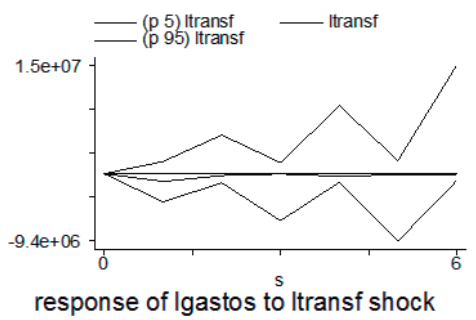

Fonte: Elaboração própria. 
Para identificar o efeito geral de choques na arrecadação, no PIB, na população e nas transferências sobre os choques nos gastos das prefeituras brasileiras, concentrar-se-á a análise dos resultados da função impulso-resposta somente aos resultados que proporcionarão a análise do efeito flypaper.

Inicialmente, verificada a presença de inovações na série do gasto público, observa-se uma reação positiva sobre o seu comportamento. Contudo, os períodos seguintes caracterizam-se por um comportamento cíclico que se anula após o transcorrer de seis períodos. Esse resultado relata que os gastos governamentais são balizados em torno de um comportamento equilibrado, já que a partir de um choque, apesar dos gastos crescerem em alguns momentos, estes tendem a apresentar uma trajetória decrescente nos períodos seguintes, de forma a viabilizar sua gestão de forma prudente.

Verificou-se que a produção impacta de forma positiva e decrescente as ações do Estado na economia. Como esperado, os choques no PIB provocam uma resposta positiva sobre os gastos públicos. Tal fato remete às necessidades apresentadas pelo setor privado, dado o crescimento da atividade produtiva, de um conjunto de investimento em infraestrutura necessário à implantação de plantas de produção, escoamento da produção, facilidades de obtenção de mão de obra e matéria-prima, etc. Portanto, a elevação do nível de atividade, caracterizada pelo aumento do PIB, requer um aumento dos gastos do governo de forma a garantir a expansão contínua da produção.

No que diz respeito à relação entre as variáveis fiscais, a função impulso-resposta apresentou uma relação negativa no momento inicial, o qual foi seguido de uma relação positiva, que apresentou uma queda acentuada nos dois períodos seguinte e que, apesar de retomar uma trajetória crescente, tornou a caracterizar-se de forma decrescente. Esse resultado é condizente com a estrutura de formação do orçamento no Estado brasileiro. As decisões orçamentárias de um período são formadas a partir da arrecadação realizada no ano anterior. Assim, espera-se que inovações na arrecadação sobre os gastos do governo sofra a defasagem de um período e se caracterize de forma positiva, ou seja, que os referidos gastos sofram um impacto positivo dos choques provenientes da arrecadação.

A relação população e gastos municipais apresentou uma função impulso-resposta com valores positivos para todos os momentos, sendo crescente até o segundo período e decrescente nos demais. Como relata Turnbull (1992), jurisdições com níveis elevados de população são condizentes com uma demanda por serviços públicos mais amplos. Assim, como localidades detentoras de um alto contingente populacional requerem um maior volume de gastos para atender suas necessidades, tem-se que um choque proveniente da população provoca o crescimento dos gastos realizados pelas prefeituras. 
Finalmente, as inovações na variável transferência produzem uma resposta negativa e decrescente sobre os gastos do governo. Assim, observa-se que choques nos repasses constitucionais promovem variações nos gastos numa magnitude inferior ao volume repassado. Esse resultado não condiz com o que a literatura denomina de efeito flypaper. Nesse mesmo sentido, Cóssio (1998), Nascimento (2010) e Mattos et al. (2011) destacam que a definição do efeito flypaper não condiz com a realidade dos municípios brasileiros.

A não verificação do efeito flypaper para o período analisado é reforçada pela comparação dos resultados observados para o impacto do PIB e das transferências sobre os gastos. Como a respostas dessa última variável em relação ao PIB é superior àquela observado para as transferências, tem-se mais uma condição contra a aceitação da hipótese do efeito flypaper.

Apresentado o efeito geral das transferências constitucionais sobre os gastos prefeituras brasileiras, que subsidiaram a análise do efeito flypaper, passa-se ao relato do modelo de regressões quantílicas para dados em painel, o qual destaca o comportamento dos gastos públicos para as esferas inferiores de governo ao longo de toda sua distribuição. A Tabela 4 destaca os resultados das estimações do modelo para os gastos governamentais.

Tabela 4 - Estimativas do modelo para os gastos municipais através de regressões quantílicas para dados em painel

\begin{tabular}{l|c|c|c|c|c}
\hline \multicolumn{7}{c}{ Variável dependente: gastos totais } \\
\hline \multicolumn{1}{c}{ Variável explicativa } & $\mathbf{0 . 1 0}$ & $\mathbf{0 . 2 5}$ & $\mathbf{0 . 5 0}$ & $\mathbf{0 . 7 5}$ & $\mathbf{0 . 9 0}$ \\
\hline \multirow{2}{*}{ Constante } & 207623.63 & 185012.29 & 196850.47 & 246743.80 & $300366.87^{*}$ \\
& $(108623.36)$ & $(103309.81)$ & $(125948.47)$ & $(120240.58)$ & $(83163.33)$ \\
\hline \multirow{2}{*}{ População } & $120.49^{*}$ & $114.19^{*}$ & $129.79^{*}$ & $159.89^{*}$ & $194.29^{*}$ \\
& $(15.11089)$ & $(15.87)$ & $(14.92)$ & $(16.10)$ & $(20.61)$ \\
\hline PIB & $6.81^{*}(1.55)$ & $6.44^{*}(1.40)$ & $5.83^{*}(2.03)$ & $6.18^{*}(2.20)$ & $11.36^{*}(2.00)$ \\
\hline Transferências & $0.31^{*}(0.02)$ & $0.39^{*}(0.01)$ & $0.44^{*}(0.01)$ & $0.45^{*}(0.01)$ & $0.44^{*}(0.01)$ \\
\hline \multirow{2}{*}{ Arrecadação } & -6.04 & -4.86 & -2.46 & 0.87 & -11.24 \\
& $(4.07)$ & $(4.97)$ & $(6.79)$ & $(7.60)$ & $(7.88)$ \\
\hline
\end{tabular}

Fonte: Elaboração própria.

Nota: Os valores entre parênteses referem-se aos erros padrão das estimativas obtido via Bootstrap; * significativo a $5 \%$.

Tendo como referência o nível de significância de 5\% para os valores críticos da estatística $t$, verifica-se que somente para a variável arrecadação não é possível rejeitar a hipótese nula, ou seja, as estimativas para os parâmetros da referida variável apresentou um efeito marginal nulo. Ainda, no que se refere à estimativa da constante, observa-se que o último dos quantis foi o único que se apresentou de forma significativa.

O primeiro desses resultados destaca a ausência do efeito da arrecadação própria sobre os gastos públicos. As finanças dos municípios brasileiros são formadas praticamente de transferências das esferas superiores de governo, tendo 
o Fundo de Participação dos Municípios, pelo governo federal, e os repasses de ICMS e IPVA, pelo governo do estado. O último retrata que a média dos gastos do governo é nula quando as variáveis população, PIB, transferência e arrecadação própria são iguais a zero, caracterizando a forte dependência das prefeituras em relação à atividade econômica e aos financiamentos das outras esferas do governo.

Os sentidos das relações mostraram-se condizentes com a literatura, com exceção do efeito negativo da arrecadação sobre os gastos. Verificou-se um efeito positivo da população e do PIB, indicando que um maior contingente populacional e um alto nível de atividade econômica requerem um aumento na provisão de bens pelo setor público, da mesma forma que um aumento nos repasses governamentais proporciona uma elevação das receitas municipais e, consequentemente, cria condições para o aumento das despesas locais. Apesar do sentido negativo encontrado para a relação entre arrecadação e gastos, como as estimativas para os parâmetros ligados à primeira dessas variáveis mostraram-se insignificantes, então o resultado para tal associação não condiz com uma negação da teoria, mas sim que nesse caso o efeito observado foi nulo. Outra justificativa pode ser obtida no fato da arrecadação própria corresponder, em média, a menos de $10 \%$ do volume de receitas municipais, fazendo com que a exploração de sua base individual não tenha grande relevância para a determinação das despesas realizadas.

Segundo o modelo do eleitor mediano, as decisões dos indivíduos são divididas entre o consumo de bens públicos e privados. Logo, para um dado aumento da população, observa-se um crescimento da demanda, e, consequentemente, a realização de novos gastos pelas prefeituras para suprir a necessidades locais se fazem necessários. As estimações mostraram que o impacto marginal de tal fato é decrescente do primeiro para o segundo quantil e crescente ao longo dos demais estratos, caracterizando que os gastos nos maiores municípios brasileiros sofrem uma maior influência de variações no tamanho da população. Cóssio e Carvalho (2001) destacam o efeito heterogêneo de flutuações no contingente populacional sobre as gastos dos municípios no Brasil. Seus resultados destacam que os municípios da região Sul e Sudeste possuem uma flutuação nos gastos superior à observada para os municípios do Nordeste e Centro-Oeste, dada uma variação no número de habitantes. Cóssio (1998) também retrata as diferentes elasticidades gasto-população existentes nas jurisdições brasileiras, identificando que as capitais brasileiras possuem uma elasticidade superior em 10\% à observada para os municípios do interior.

Os efeitos da produção sobre os gastos do governo assumiram um comportamento inicialmente decrescente, mas seguido de valores crescente nos últimos quantis. Esse resultado caracteriza o efeito marginal da referida variável num forma de "U", retratando que aqueles municípios com os menores e maiores gastos 
sofrem maior influência de flutuações no PIB do que aqueles em quantis intermediários. Ainda, cabe destacar que o multiplicador dos gastos é superior, assim como para variável população, naquelas jurisdições que possuem uma maior atuação do governo, ou seja, nas municipalidades com maiores despesas municipais.

Como esperado, a flutuação no PIB impacta positivamente nos gastos das prefeituras brasileiras. Tal fato deve-se à necessidade de formação de infraestrutura necessária ao desenvolvimento da atividade produtiva. A concentração da produção em determinadas localidades requer a instalação de: centros formadores de mão de obra; estradas, ferrovias, portos e aeroportos para escoamento da produção, bem como para facilitar o fornecimento de matérias-primas indispensáveis às atividades; centros de coleta para resíduos industriais; condições sanitárias adequadas; uma rede de serviços ambulatórias e postos de saúde para prevenção e acompanhamento de enfermidades, etc.

Por outro lado, é plausível supor que um dado aumento na renda faça com que a população local demande mais bens e serviços governamentais como apresentado por Turnbull (1992), e, assim, se faça necessária uma elevação dos gastos públicos de modo a oferecer a devida quantidade requerida na cesta da população, justificando um impacto positivo e significativo do PIB sobre as despesas municipais.

No que diz respeito à relação entre o gasto total e as transferências intergovernamentais, identificou-se um efeito marginal positivo e inferior ao observado para a variável PIB. Esse resultado estabelece que, para uma dada variação nos repasses constitucionais, verifique-se uma flutuação no comportamento dos gastos municipais de formar menos expressiva, ou seja, para cada real adicional repassado tem-se um aumento nos gastos entre 0,31 e 0,45 centavos de real, sendo essa relação crescente até o quarto quantil e com uma leve redução no último quantil. Ainda, já que o referido efeito das transferências sobre as despesas mostrou-se inferior ao observado para o PIB, tem-se que o comportamento dos gastos públicos das prefeituras brasileiras não condiz com a definição de uma postura imprudente denunciada pela literatura como efeito flypaper. Esse resultado contradiz o exposto por Cóssio e Carvalho (2001), que verificam a prática insensata de variações nos gastos superiores ao volume de receitas provenientes de repasses constitucionais, provavelmente por tratarem a discussão através de uma estrutura em cross-section de mais de 3.500 municípios brasileiros para o ano de 1996.

Apesar de o modelo PVAR(1) apresentar um sentido negativo para a relação gastos-transferências e o modelo de regressões quantílicas em painel verificar um movimento oposto, esses resultados não apresentam contradições. A PVAR destaca o comportamento dinâmico para a referida relação, caracterizando de forma negativa a resposta dos gastos aos choques nas transferências, evidenciando que as prefeituras brasileiras não assumem uma postura fiscal indisciplinada ao longo 
do tempo, ou seja, não são realizados gastos superiores ao volume de receitas de forma sucessiva. O modelo de regressões quantílicas, que trata tal relação de forma estática, relata que o comportamento irracional de elevar os gastos de forma acentuada e superior aos repasses, que são sua principal fonte de receita, produzindo déficits orçamentários de forma progressiva, não se faz presente ao longo dos quantis trabalhados, principalmente para os primeiros estratos. Portanto, não existem indícios do efeito flypaper na análise geral fornecida pelo PVAR, ou ao longo da distribuição como predita pelo modelo quantílico.

\section{Considerações Finais}

Este trabalho teve como foco a análise do comportamento das despesas dos municípios brasileiros mediante o sistema de transferências intergovernamentais que propiciam sua principal fonte de receitas. Através das técnicas de vetores autorregressivos e de regressões quantílicas, ambas em suas respectivas versões para dados em painel, propostas por Holtz (1988) e Koenker (2004), respectivamente, foram visualizados os efeitos dos referidos repasses sobre os gastos de 5.293 municípios, entre 1999 e 2009.

A observação de uma prática imprudente na qual os gestores promovem uma variação nos gastos superior quando da ocorrência de choques nas transferências em relação àqueles provenientes de inovações na produção não pode ser admitida para as administrações dos municípios brasileiros. Como destacado anteriormente, quando as decisões dos agentes são formadas perante um contexto de incerteza, não é possível identificar condições que caracterizam a ação expressa pelo efeito flypaper. Tal fato é justificado através do efeito negativo de inovações na série das transferências sobre as flutuações da despesa pública apresentadas pela função impulso-resposta.

Cabe destacar a influência positiva de choques no PIB, na população e na arrecadação sobre a série da execução orçamentária realizadas pelas prefeituras brasileiras entre 1999 e 2009. Como previsto inicialmente, o aumento da demanda via consumo e investimento privado, bem como o crescimento das receitas observadas por choques positivos na arrecadação promovem a elevação das despesas na administração municipal.

O complemento para a análise trabalhada pelo modelo de vetores autorregressivos para dados em painel foi idealizada pela técnica de regressões quantílicas proposta por Koenker (2004), que possibilita a verificação dos efeitos ao longo de toda a distribuição.

Por um lado, o tratamento via quantis possibilitou visualizar que os efeitos da renda superam àqueles observados para as transferências ao longo dos quantis, 
e, portanto, não se tem indícios de que a definição de flypaper é observada pela gestão municipal praticada no Brasil.

A análise por quantis revelou, ainda, uma relação positiva e crescente da produção e da população sobre as despesas municipais ao longo dos quantis. De fato, um maior número de indivíduos residentes numa jurisdição, aliado ao crescimento da atividade econômica, só poderia trazer consequências positivas sobre a atuação do setor público no que diz respeito à provisão de serviços essenciais, infraestrutura, etc.

Por fim, pode-se identificar que não existem diferenças nos sentidos das relações observadas pelo PVAR e pelo modelo de regressões quantílicas para dados em painel, sendo somente verificada uma distinção nas magnitudes, ou seja, a análise geral chega às mesmas conclusões obtidas em diferentes pontos da distribuição, apesar das diferentes proporções observadas ao longo dos quantis. Portanto, dado que não foram identificadas contradições nos sentidos das relações, no que tange aos resultados de ambas as técnicas, não se verificou condições que comprovem que o efeito flypaper é uma evidência para a realidade brasileira tanto do ponto de vista geral, quanto para o caso particular.

\section{Referências}

BACARREZA-CANAVIRE, G.; ZUÑIGA ESPINOZA, N. G. Fiscal transfers a curse or blessing? Evidence of their effect on tax effort for municipalities in Sinaloa, Mexico. Atlanta, Georgia: Georgia State University, 2010. (International Studies Program Working Paper, n. 10-30).

BINDER, M.; HISAO, C.; PESSARAM, M. H. Estimation and inference in short panel vector autoregressions with unit roots and cointegration. Econometric Theory, v. 21, n. 4, p. 795-837, 2004.

BREITUNG, J. The local power of some unit root tests for panel data. In: BADI, H. et al. (Ed.). Nonstationary panels, panel cointegration, and dynamic panels. Amsterdam: JAI Press, 2001. (Advances in Econometrics, v. 15). p. 161-178.

BREITUNG, J.; DAS, S. Panel unit root tests under cross-sectional dependence. Statistica Neerlandica, v. 59, n. 4, p. 414-433, Nov. 2005.

CHOI, I. Unit root tests for panel data. Journal of International Money and Finance, v. 20, n. 2 , p. 249-272, Apr. 2005.

COSSÍO, F. A. B. Disparidades econômicas inter-regionais, capacidade de obtenção de recursos tributários, esforço fiscal e gasto público no federalismo brasileiro. 1998. 134 f. Dissertação (Mestrado em Economia) - Pontifícia Universidade Católica do Rio de Janeiro, Rio de Janeiro, 1998. Disponível em: <http://www.bndes.gov.br/SiteBNDES/export/sites/default/ bndes_pt/Galerias/Arquivos/conhecimento/premio/pr211.pdf >. Acesso em: 26 jun. 2003.

COSSíO, F. A. B.; CARVALHO, L. M. Os efeitos expansivos das transferências intergovernamentais e transbordamentos espaciais de despesas públicas: evidências para os municípios brasileiros - 1996. Pesquisa e Planejamento Econômico, v. 31, n. 1, p. 75-124, 2001. 
COURANT, P. N.; GRAMLICH, E.; RUBINFIELD, D. The stimulative effects of intergovernmental grants: or why money sticks where it hits. In: MIESZKOWSKI, P.; OAKLAND, W. H. (Ed.). Fiscal federalism and grants-in-aid. Washington, D.C.: The Urban Institute, 1979.

FILIMON, R.; ROMER, T.; ROSENTHAL, H. Asymmetric information and agenda control: the bases of monopoly power in public spending. Journal of Public Economics, v. 17, n. 1, p. 51-71, 1982.

FISHER, R. C. Income and grants effects on local expenditure: the flypaper effect and other difficulties. Journal of Public Economics, v. 17, n. 3, p. 51-70, 1982.

GANG, N.; KHAN, H. Foreign aid and fiscal behavior in a bounded rationality model: different policy regimes. Empirical Economics, v. 24, n. 1, p. 121-134, 1999.

GRAMLICH, E. Intergovernmental grants: a review of the empirical literature. In: OATES, W. (Ed.). The political economy of fiscal federalism. New York: Lexington Press, 1977.

GRAMLICH, E.; GALPER, H. State and local fiscal behavior and federal grant policy. Brookings Papers on Economic Activity, v. 4, n. 1, p. 15-58, 1973.

HADRI, K. Testing for stationarity in heterogeneous panel data. Econometrics Journal, v. 3, n. 2, p. 148-161, Dec. 2000.

HARRIS, R. D. F.; TZAVALIS, E. Inference for unit roots in dynamic panels where the time dimension is fixed. Journal of Econometrics, v. 91, n. 2, p. 201-226, Aug. 1999.

HELLER, P. S. A model of public fiscal behavior in developing countries: aid, investment and taxation. American Economic Review, v.3, n. 65, p. 429-445, June 1975.

HOLTZ-EAKIN, D.; NEWEY, W.; ROSEN, H. S. Estimating vector autoregressions with panel data. Econometrica: Journal of the Econometric Society, v. 6, n. 56, p. 1371-1395, Nov. 1988.

IBGE. Base de Informações Municipais - BIM. 2010. Disponível em: < http://www.ibge.gov.br/ home/estatistica/economia/perfilmunic >. Acesso em: 26 jun. 2011.

IM, K. S.; PESARAN, M. H.; Y. SHIN. Testing for unit roots in heterogeneous panels. Journal of Econometrics, v. 115, n. 1, p. 53-74, July 2003.

IQBAL, Z. Foreign aid and the public sector: a model of fiscal behavior in Pakistan. The Pakistan Development Review, v. 36, n. 2, p. 115-129, 1997.

KHAN, H.; HOSHINO, E. Impact of foreign aid on the fiscal behaviour of LDC governments. World Development, v. 20, n. 10, p. 1481-1488, Oct. 1992.

KHILJI, N. M.; ZAMPELLI, E. M. The fungibility of US assistance to developing countries and the impact on recipient expanditures: a case study of Pakistan. Elsevier, v. 19, n. 8, p. 10951105, Aug. 1991.

KOENKER, R. Quantile regression for longitudinal data. Journal of Multivariable Analysis, v. 91, n. 1, p. 74-89, Oct. 2004. 
KOENKER, R.; BASSETT, G. Regression quantile. Econometrica, v. 46, n.1, p. 33-50, Jan. 1978.

LEVIN, A.; LIN, C. F.; CHU, C. S. J. Unit root tests in panel data: asymptotic and finite-sample properties. Journal of Econometrics, v. 108, n. 1, p. 1-24, May 2002.

LOVE, I.; ZICCHINO, L. Financial development and dynamic investment behavior: evidence from panel VAR. The Quarterly Review of Economics and Finance, v. 46, n. 2, p. 190-210, 2006.

MATTOS, E.; ROCHA, F.; ARVATE, P. Flypaper effect revisited: evidence for tax collection efficiency in Brazilian municipalities. Estudos Econômicos, v. 41, n. 2, p. 239-267, 2011.

MCGUIRE, M. C. A method for estimating the effects of a subsidy on the receiver's resource constraint: with an application to the U.S. local government, 1964-1971. Journal of Public Economics, v. 10, n. 1, p. 25-44, Aug. 1978.

MORENO, L. C. Fiscal performance of local governments in México: the role of Federal Transfers. Mexico: Centro de Investigación y Docencia Económica, 2003. (Working Paper, n. 127).

MOSLEY, P.; HUDSON, J.; HORRELL, S. Aid, the public sector and the market in less developed countries. The Economic Journal, v. 97, n. 387, p. 616-641, Sept. 1987.

NASCIMENTO, J. S. Efeitos das transferências financeiras sobre o gasto e a arrecadação dos municípios brasileiros. 2010. 159 f. Tese (Doutorado em Economia Aplicada) - Universidade de Viçosa, Viçosa, MG, 2010.

OATES, W. E. Fiscal Federalism. New York: Harcourt Brace Jovanovich, 1972.

. Lump sum intergovernmental grants have price effects. In: MIESZKOWSKI, P.; OAKLAND, W. H. (Ed.). Fiscal federalism and grants-in-aid. New Yorl: The Urban Institute, 1979.

ORAIR, R.; ALENCAR, A. Esforço fiscal dos municípios: indicadores de condicionalidade para o sistema de transferências intergovernamentais. Brasília, DF: ESAF, 2010. 60 p. Monografia premiada em $1^{\circ}$ lugar no XV Prêmio Tesouro Nacional, Tópicos Especiais de Finanças Públicas.

PACK, H.; PACK, J. R. Foreign aid and the question of fungibility. The Review of Economics and Statistics, v. 75, n. 3, p. 258-265, 1993.

RIBEIRO, E. P. Capacidade e esforço tributário no Rio Grande do Sul: o caso dos municípios. Perspectiva Econômica On Line, São Leopoldo, v. 1, n. 1, p. 21-49, jan./jun. 2005.

RIBEIRO, E. P. Transferências intergovernamentais e esforço fiscal dos estados brasileiros. In: ENCONTRO BRASILEIRO DE ECONOMETRIA, 20., 1998, Vitória. Anais... Vitória: Sociedade Brasileira de Econometria, 1998.

RIBEIRO, E. P.; SHIKIDA, C. D. Existe trade-off entre receitas próprias e transferências? O caso dos municípios mineiros. In: SEMINÁRIO SOBRE A ECONOMIA MINEIRA, 9., 2000, Diamantina, MG. Anais... Diamantina, MG: Cedeplar/UFMG, 2000. 
SCHWENGBER, S. B.; RIBEIRO, E. P. E. O Impacto do Fundo de Participação (FPE) no esforço tributário dos Estados: uma estimativa do potencial de arrecadação do ICMS. Brasília, DF: Esaf, 2000. (IV Prêmio Tesouro Nacional - coletânea de monografias).

STRUMPF, K. S. A predictive index for the flypaper effect. Journal of Public Economics, v. 69, n. 3, p. 389-412, 1998.

TURNBULL, G. Fiscal illusion, uncertainty, and the flypaper effect. Journal of Public Economics, v.48, n. 2, p. 207-223, 1992.

WHITE, H. Foreign aid, taxes and public investment: a further comment. Journal of Development Economics, v. 45, n. 1, p. 155-163, Oct. 1994.

ZHANG, H.; HU, W. Impact of fiscal transfer of effort of Chinese provincial government. Frontiers of Economics in China, v. 4, n.3, p. 406-424, Sept. 2009.

Recebido em: 16/10/2014. Aceito em: 12/02/2015. 